\title{
OPEN Chronic wasting disease (CWD) prion detection in blood from pre-symptomatic white-tailed deer harboring PRNP polymorphic variants
}

\author{
Carlos Kramm ${ }^{1,2}$, Paulina Soto ${ }^{1}$, Tracy A. Nichols ${ }^{3}$ \& Rodrigo Morales ${ }^{1,4 凶}$
}

Chronic wasting disease (CWD) is a prionopathy affecting wild and farmed cervids. This disease is endemic in North America and has been recently identified in Europe. Ante-mortem CWD tests of preclinical cervids may be an important tool in helping control the spread of this disease. Unfortunately, current CWD diagnostic methods are not suitable for non-tissue type samples. We reported that CWD prions can be detected in blood of pre-clinical CWD-infected white-tailed deer (WTD) with high sensitivity and specificity using the Protein Misfolding Cyclic Amplification (PMCA) assay. However, that report only included animals homozygous for codon $96 \mathrm{G}$, the most common polymorphic version of the prion protein within this animal species. Here, we report CWD prion detection using blood of naturally infected WTD coding one or two copies of the PrP-96S polymorphic variant. Our results, from a blinded screening, show $100 \%$ specificity and $~ 58 \%$ sensitivity for animals harboring one $96 \mathrm{~S}$ codon, regardless of their stage within the pre-clinical phase. Detection efficiency for PrP-96S homozygous animals was substantially lower, suggesting that this allele affect peripheral prion replication/tropism. These results provide additional information on the influence of codon 96 polymorphisms and the ability of PMCA to detect CWD in the blood of pre-clinical WTD.

Chronic wasting disease (CWD) is the only prion disease found in free-ranging animals ${ }^{1-3}$. Screening and containment programs aimed at halting CWD spread have been established with variable success ${ }^{4-6}$. Unfortunately, the lack of practical diagnostic methods has been one of the factors stopping the implementation of more efficient containment practices. At present, the gold-standard method for CWD diagnosis is the immunohistochemical (IHC) analysis of disease-associated prion protein $\left(\mathrm{Pr}^{\mathrm{Sc}}\right)$ accumulation in medial retropharyngeal lymph nodes and obex (https://www.aphis.usda.gov/animal_health/animal_diseases/cwd/downloads/cwd-program-stand ards.pdf). The fact that several bodily fluids and excreta from CWD infected animals carry prion infectivity ${ }^{7-9}$ provides potential targets for animal diagnostic testing. Our lab and others have shown that in vitro prion replication techniques (such as the protein misfolding cyclic amplification (PMCA) ${ }^{10}$ and real-time quaking induced conversion (RT-QuIC) ${ }^{11}$ ) have the potential for use in pre-clinical diagnosis using a wide variety of biological samples. In a previous publication ${ }^{12}$, we communicated the results of CWD prion screening using blood from a group of farm raised pre-symptomatic white-tailed deer (WTD). Our results showed $96 \%$ sensitivity at late pre-symptomatic stages, and $53 \%$ at early stages. The specificity of our assay was $100 \%$. These results suggested that PMCA can identify a fraction of CWD positive animals.

A drawback from the study mentioned above is that data was collected using specimens from animals harboring the most common prion protein $\left(\mathrm{PrP}^{\mathrm{C}}\right)$ sequence only. $\mathrm{PrP}^{\mathrm{C}}$ polymorphisms are seen in several animal species, including cervids ${ }^{2,13-16}$. In WTD, the most commonly found polymorphism occurs at position 96 where a glycine $(G)$ is substituted for a serine $(S)^{17}$. The presence of at least one $S$ at position 96 is associated with longer incubation periods compared to animals expressing the most common ("wild-type", PrP 96GG) genotype g $^{17,18}$.

\footnotetext{
${ }^{1}$ Department of Neurology, McGovern Medical School, The University of Texas Health Science Center at Houston, Houston, TX 77030, USA. ²Facultad de Medicina, Universidad de Los Andes, Las Condes, Av. San Carlos de Apoquindo 2200, Santiago, Chile. ${ }^{3}$ United States Department of Agriculture, Animal Plant Health Inspection Service, Veterinary Services, Fort Collins, CO 80526, USA. ${ }^{4} \mathrm{CIBOA}$, Universidad Bernardo O'Higgins, Santiago, Chile. ${ }^{\circledR}$ email: Rodrigo.MoralesLoyola@uth.tmc.edu
} 


\begin{tabular}{|l|l|l|l|l|l|l|l|}
\hline Animal ID & $\begin{array}{l}\text { CWD status by } \\
\text { IHC }\end{array}$ & $\begin{array}{l}\text { PrP (at position } \\
\text { 96) }\end{array}$ & PMCA result & Animal ID & $\begin{array}{l}\text { CWD status by } \\
\text { IHC }\end{array}$ & $\begin{array}{l}\text { PrP (at position } \\
\text { 96) }\end{array}$ & PMCA result \\
\hline 037 & Obex/RPLN & GS & + & 163 & RPLN & GS & + \\
\hline 053 & Obex/RPLN & GS & + & 181 & RPLN & GS & + \\
\hline 063 & Obex/RPL & GS & - & 184 & RPLN & GS & + \\
\hline 064 & Obex/RPL & GS & - & 217 & RPLN & GS & - \\
\hline 065 & Obex/RPLN & GS & + & 287 & RPLN & GS & - \\
\hline 077 & Obex/RPLN & GS & + & 029 & nd & GS & - \\
\hline 111 & Obex/RPLN & GS & - & 043 & nd & GS & - \\
\hline 123 & Obex/RPLN & GS & - & 054 & nd & GS & - \\
\hline 129 & Obex/RPLN & GS & + & 074 & nd & GS & - \\
\hline 134 & Obex/RPLN & GS & + & 083 & nd & GS & - \\
\hline 142 & Obex/RPLN & GS & - & 091 & nd & GS & - \\
\hline 166 & Obex/RPLN & GS & + & 093 & nd & GS & - \\
\hline 040 & RPL & GS & + & 270 & nd & GS & - \\
\hline 042 & RPLN & GS & - & 085 & RPLN & SS & - \\
\hline 080 & RPLN & GS & - & 094 & RPLN & SS & - \\
\hline 087 & RPLN & GS & - & 116 & RPLN & SS & - \\
\hline 092 & RPLN & GS & - & 159 & RPLN & SS & + \\
\hline 108 & RPLN & GS & + & 160 & RPLN & SS & - \\
\hline 113 & RPLN & GS & - & 089 & nd & SS & - \\
\hline 133 & RPLN & GS & - & 100 & nd & SS & - \\
\hline 140 & RPLN & GS & + & 109 & nd & SS & - \\
\hline & & & & & & \\
\hline
\end{tabular}

Table 1. Individual data of white tail deer used in this study. ${ }^{\star}$ Indicates positive detection of PK resistant $\operatorname{PrP}$ in Obex or medial retropharyngeal lymph node (RPLN) tissues by immunohistochemistry (nd: non detected).

Importantly, animals carrying PrP 96S allele(s) have been associated with lower prion shedding, suggesting lower accumulation of $\mathrm{PrP}^{\mathrm{Sc}}$ in peripheral tissues compared to their wild-type counterpart ${ }^{19,20}$. This variable could have important implications for pre-clinical diagnosis when using peripheral tissue biopsies, bodily fluids and excreta. In this work, we evaluated the diagnostic efficiency of the PMCA technology using blood specimens collected from farmed/non-symptomatic WTD carrying one or two PrP 96S allele(s).

\section{Materials and methods}

Ethics statement. Blood samples from male and female WTD were collected from farmed herds depopulated due to CWD by the USDA. These samples were sent to Dr. Morales' group for prion screening using the PMCA technology. The investigators at Dr. Morales' lab were blinded to the identity of the samples when performing the PMCA assay. All work manipulating these samples was previously approved by the Institutional Biosafety Committee of The University of Texas Health Science Center at Houston (UTHealth). PMCA substrate was derived from brains of transgenic mice expressing the WTD prion protein expressing glycine at position 96 $\left(\operatorname{Tg} 1536^{21}\right)$. Procedures for animal handling were approved by the Institutional Animal Care and Use Committee at UTHealth.

Study populations. The animal population included subjects coding for the $96 \mathrm{~S}$ polymorphic variant of the prion protein ( $81 \%$ heterozygous, $19 \%$ homozygous). All animals were devoid of classical CWD clinical signs (including but not limited to neurological abnormalities, polydipsia, polyuria, weight loss, etc.) as assessed by Dr. Tracy Nichols and/or other qualified USDA personnel. These animals were sacrificed immediately after blood collection. CWD status was assessed by the presence of protease-resistant PrP aggregates by IHC in the obex and retropharyngeal lymph nodes (RPLN). CWD staging was assigned as follows: obex (+) and RPLN (+): late pre-symptomatic; obex (-) and RPLN (+): early pre-symptomatic; obex (-) and RPLN (-): non-detect. In summary, blood samples from 42 animals (12 late pre-symptomatic, 19 early pre-symptomatic, and 11 CWDnon detect) were used in this study. The specific data for blood donors is displayed in Table 1.

Blood sample collection. Blood was collected from the jugular vein of chemically immobilized animals using commercially available blood collection tubes containing EDTA as anticoagulant. Specimens were frozen and stored at $-80{ }^{\circ} \mathrm{C}$ at USDA facilities (Fort Collins, CO). These samples were overnight-transferred to UTHealth in containers filled with ice packs. Once at UTHealth, samples were stored again at $-80^{\circ} \mathrm{C}$ until use.

Sample processing. Blood specimens were thawed at room temperature and vortexed. One aliquot $(10 \mu \mathrm{L})$ of each of these samples was directly mixed with PMCA substrate, while a second aliquot $(200 \mu \mathrm{L})$ was processed for $\mathrm{PrP}^{\mathrm{Sc}}$ concentration by ultracentrifugation as previously described ${ }^{10,12,22}$. Briefly, the $200 \mu \mathrm{L}$ blood aliquots were mixed with equivalent volumes of a $20 \% \mathrm{w} / \mathrm{v}$ sarkosyl solution (prepared in PBS) for $1 \mathrm{~h}$ at room tempera- 


\section{Seed GG}

\section{PMCA Round 1}

A)

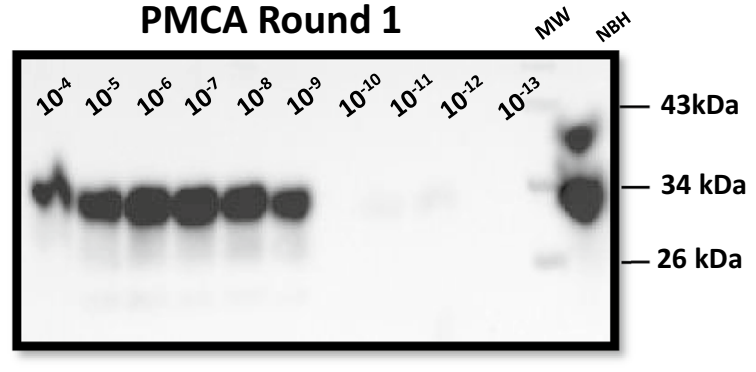

Seed GS

B)

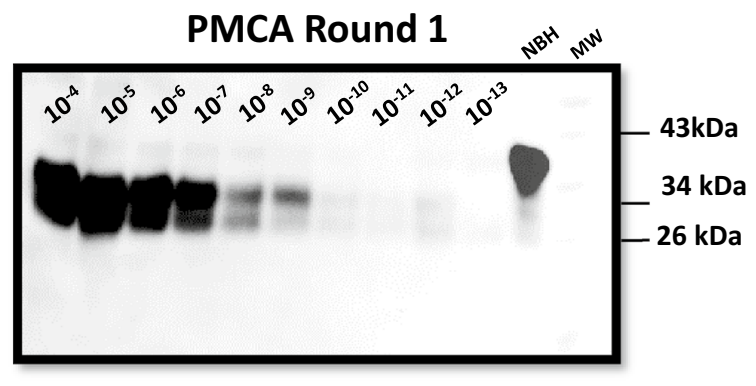

Seed GG

PMCA Round 3

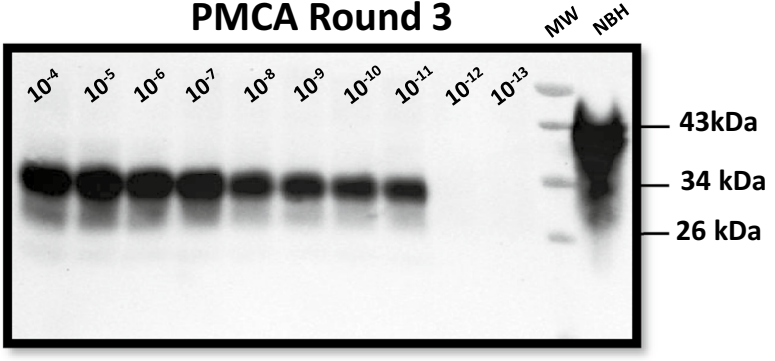

Seed GS

PMCA Round 3

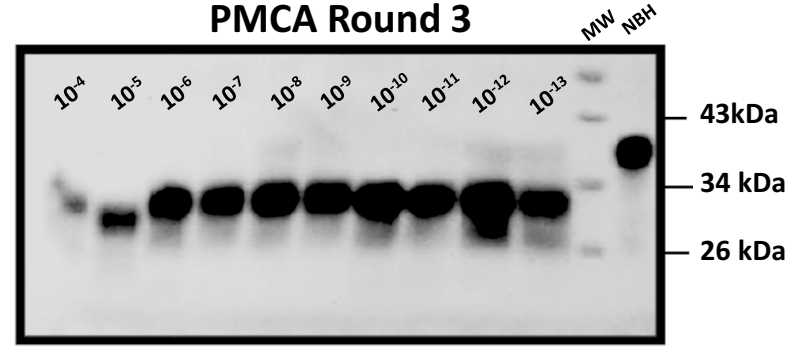

Figure 1. In vitro prion replication of WTD brain-derived PrP 96GG and PrP 96GS CWD prions. Serial dilutions of normalized PK-resistant PrP 96GG (A) and PrP 96GS (B) CWD prions (brain-derived) were loaded into PMCA reactions and tested for their replication efficiencies. This figure depict results of the 1st (left panels) and 3rd (right panels) PMCA rounds. MW represent molecular weight protein standards. Normal Brain Homogenate $(\mathrm{NBH})$ represents non PK-treated brain extracts from Tg1536 mice used as molecular weight migration and antibody specificity controls. Numbers at the right of right panels represent molecular weight markers (in $\mathrm{KDa}$ ).

ture and then centrifuged at $100,000 \times g$ for $1 \mathrm{~h}$ at $4{ }^{\circ} \mathrm{C}$. Supernatants were carefully discarded without disturbing pellets, $400 \mu \mathrm{L}$ of PBS added to them, and centrifuged using the same protocol described above but for just $30 \mathrm{~min}$. Resulting pellets were resuspended in PMCA conversion buffer and transferred to $200 \mu \mathrm{L}$ PCR tubes.

PMCA assay and western blotting. The PMCA procedure was performed as described previously ${ }^{10,12,23}$. Briefly, each blood sample was run in quadruplicates considering the modalities explained above: direct spiking and concentration (samples run twice for each modality). PMCA products were treated with $100 \mu \mathrm{g} / \mathrm{mL}$ proteinase $\mathrm{K}(\mathrm{PK})$ at $37^{\circ} \mathrm{C}$ and constant shaking for $1 \mathrm{~h}$. PK reaction was stopped by adding LDS sample buffer and exposure to $95^{\circ} \mathrm{C}$ for $10 \mathrm{~min}$. Later, samples were fractionated by SDS-PAGE and transferred to nitrocellulose membranes as described ${ }^{10}$. Membranes were probed with either 6H4 (Fig. 1) or PRC1 (Fig. 2) antibodies at 1:10,000 or 1:5000 dilutions, respectively. Membranes were developed using a chemoluminiscence method as explained in previous publications ${ }^{10,22,24}$. A blood sample was considered as CWD positive if at least one of the replicates exhibited PK resistant PMCA products, as previously described ${ }^{12}$. Readouts were taken at the 4 th PMCA round.

Statistical analysis. Overall correct diagnosis was calculated using the following formula: (correct PMCA diagnosis in both positive and negative groups/total number of cases) $\times 100$. Estimated predictive values for sensitivity and specificity were calculated using the MedCalc software (https://www.medcalc.org/calc/diagnostic _test.php).

Ethical approval. The experiments listed in this manuscript did not involved animal experimentations. Blood samples from WTD were collected previously for other purposes by TN. We have USDA approval to receive and work with these samples. As substrate for PMCA, we used brains from transgenic mice expressing deer $\operatorname{PrP}^{\mathrm{C}}$. The procedures for breeding, manipulations and euthanasia of these transgenic mice was performed following NIH guidelines and approved by the Animal Welfare Committee of The University of Texas Health Science Center at Houston (protocol AWC-17-0049).

Original western blot files. Original files for the figures included in this article can be found at Supplemental Figure S1. 

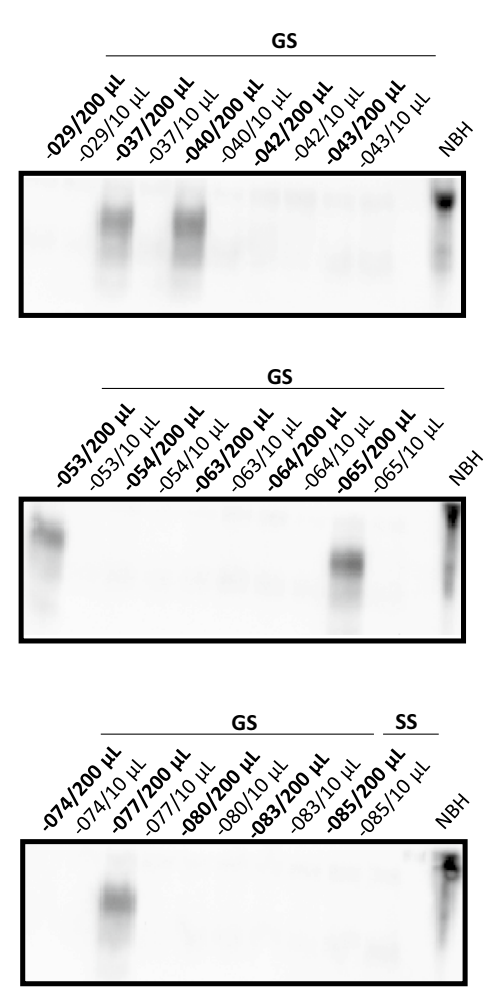
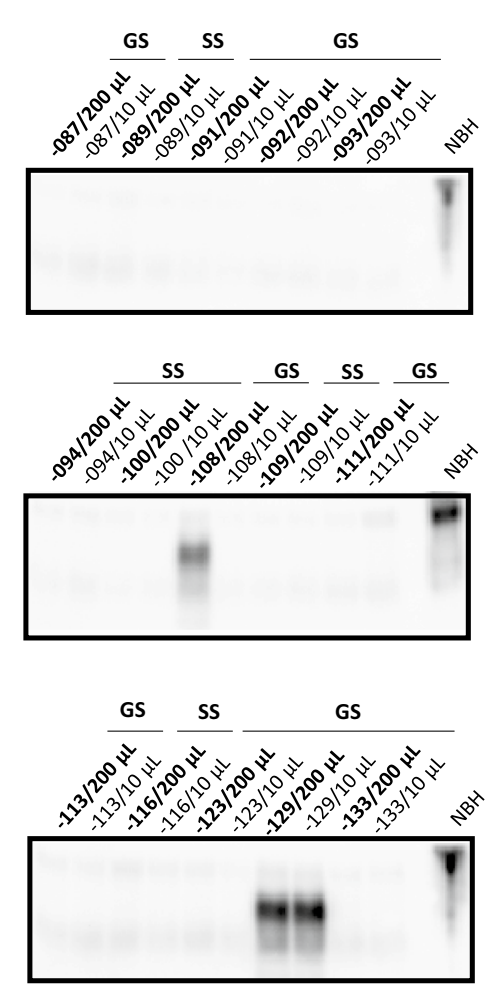
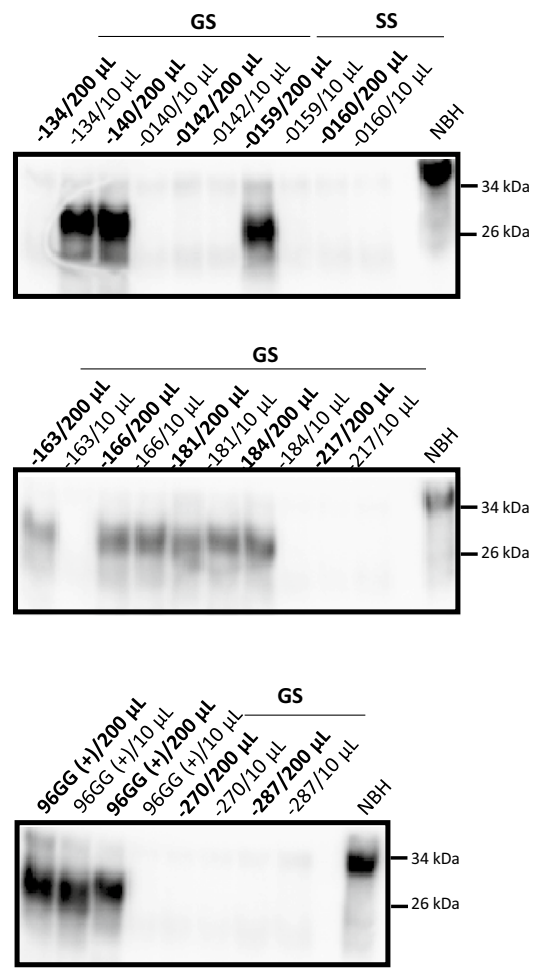

Figure 2. CWD prion detection in blood samples from WTD expressing for PrP 96S. PK-treated PMCA products from WTD-derived blood are depicted. Blood samples were collected from WTD expressing for at least one copy of the PrP 96S polymorphic variant. Blood samples were tested in PMCA by either direct spiking $(10 \mu \mathrm{L})$ or concentration $(200 \mu \mathrm{L})$ as described in Materials and Methods. "GS" or "SS" at the top of each panel depict PrP polymorphic classification. Sample ID is represented in each lane using the "-XX" format. 96GG (+) represent blood of late pre-symptomatic PrP 96GG deer used as positive control. Normal Brain Homogenate (NBH) represents non PK-treated brain extracts from Tg1536 mice used as molecular weight migration and antibody specificity controls. Numbers at the right of right panels represent molecular weight markers (in KDa). This figure depict two of the four replicates tested for each sample. Samples -080 and -092 were PMCA positive in a different set of replicates (not shown).

\section{Results}

Several reports involving diverse animal species suggest that prion transmission may be influenced by interpolymorphic prion factors ${ }^{25}$. For that reason, we first evaluated the efficiency of our previously published cervid PMCA system ${ }^{12,23}$ that utilized substrate homozygous for Glycine at codon 96 in the PRNP gene. This experiment was done as this substrate is not homologous to the PRNP polymorphisms of the WTD samples used in this study. Specifically, brain extracts from experimentally infected PrP 96GG and PrP 96GS WTD (kindly provided by Dr. Edward Hoover) were used to compare in vitro amplification rates. After normalizing the amount of PK-resistant $\mathrm{PrP}$ in both inocula by western blot, serial dilutions in PMCA substrate were performed. The PMCA reaction using the PrP 96GG inoculum provided amplification up to $1 \times 10^{-6}-1 \times 10^{-7}$ dilutions in the first PMCA round. At the third PMCA round, positive prion replication was reproducibly observed up to $1 \times 10^{-11}$ dilution for this particular inoculum (Fig. 1A). For the PrP 96GS sample, a similar amplification compared to its PrP 96GG counterpart was observed in the first PMCA round. At PMCA round three, we observed a higher amplification as PK-resistance PrP signals were observed at the $1 \times 10^{-13}$ dilution (Fig. 1B). This data shows that our current PMCA system is suitable to screen samples carrying at least one $\operatorname{PrP} 96 \mathrm{~S}$ allele. Unfortunately, a similar experiment using PrP 96SS CWD prions was not possible since we did not have access to this sample type.

Considering these results, we analyzed blood samples from CWD-infected WTD harboring at least one PrP 96S allele using the PMCA system described in Fig. 1. As detailed in Materials and Methods and Table 1, we analyzed a total of 42 blood samples collected from 31 CWD positive and 11 CWD non-detect animals with either the GS or SS genotype at PRNP codon 96. Prion infected subjects were distributed in 12 (39\%) late pre-symptomatic and 19 (61\%) early pre-symptomatic cases (Table 2). Readings taken at the 4th PMCA round confirmed the specificity of our assay as no samples from the CWD-non detect animals provided PK-resistant PrP signals (Table 2, Fig. 2). In blood samples from PrP 96GS animals, we observed 57.1\% (8/14) detection accuracy in the early pre-symptomatic CWD stage and a similar detection accuracy of $58.3 \%(7 / 12)$ in the late pre-symptomatic stage. For the PrP 96SS group, only 5 samples from animals at the early pre-symptomatic phase of the CWD incubation period were collected. Just one of these samples provided positive results in the PMCA assay (20\% of accurate detection, Table 2). Further PMCA rounds did not increase the efficiency or sensitivity of this assay, as assessed by using serially diluted brain extracts from CWD infected deer or blood samples (data 


\begin{tabular}{|l|l|l|l|}
\hline Biological sample & $\mathbf{9 6 G G}^{\mathbf{b}}$ & $\mathbf{9 6 G S}$ & $\mathbf{9 6 S S}$ \\
\hline $\begin{array}{l}\text { Late Pre-symptomatic WTD } \\
\text { (B+LN+) }\end{array}$ & $47 / 49$ & $7 / 12$ & $\mathrm{~ns}^{\mathrm{c}}$ \\
\hline $\begin{array}{l}\text { Early pre-symptomatic WTD } \\
\text { (B-LN+) }\end{array}$ & $95.9 \%$ & $58.3 \%$ & $1 / 5$ \\
\hline $\begin{array}{l}\text { Negative WTD } \\
\text { (B-LN-) }\end{array}$ & $18 / 34$ & $8 / 14$ & $20.0 \%$ \\
\hline Overall correct diagnosis $^{\mathrm{a}}$ & $52.9 \%$ & $57.1 \%$ & $0 / 3$ \\
\end{tabular}

Table 2. Summary of the results obtained in a blind study of detection of CWD prions in blood from whitetailed deer coding for different polymorphic versions of the prion protein (at position 96). ${ }^{\text {a Samples were }}$ declared positive if at least one of the two replicates performed provided a protease-resistant $\mathrm{PrP}^{\mathrm{Sc}}$ signal in the PMCA assay. ${ }^{b}$ Data from Kramm et al. ${ }^{12} .{ }^{c} n s$ no samples. ${ }^{d}$ Estimates of sensitivity for the PrP 96GG group, with 95\% confidence intervals: $67.9-86.8 \%$. ${ }^{e}$ Estimates of specificity for the PrP 96GG group, with 95\% confidence

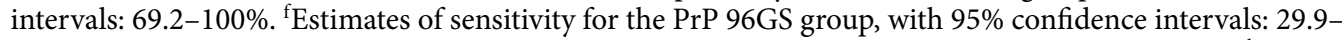
$70.1 \%$. ${ }^{\mathrm{g}}$ Estimates of specificity for the PrP 96GS group, with $95 \%$ confidence intervals: $63.1-100 \%$. ${ }^{\text {h }}$ Estimates of sensitivity for the PrP 96SS group, with 95\% confidence intervals: $0.5-71.6 \%$. ${ }^{\mathrm{i}}$ Estimates of specificity for the PrP 96SS group, with 95\% confidence intervals: $29.2-100 \%$.

not shown). Other technical modifications such as the use of different antibodies for detection in western blots (e.g., 6H4, 6D11 and PRC1) provided similar outcomes, as reported previously ${ }^{12}$.

As mentioned, each sample was tested in four replicates. An animal was considered CWD positive by PMCA if at least one of these replicates provided positive signals. Replicates consisted of (i) direct spiking of blood in PMCA substrate, or (ii) pellets derived from the concentration of larger blood volumes (Fig. 2). From the 14 PMCA positive samples in this screening, just one of them was positive in the "spiked" version only (sample - 134, Fig. 2). This modality allowed us to assess, side by side, if the concentration of large volumes of samples is advantageous compared to direct spiking of lower volumes of the same samples in the PMCA reaction. As expected, our results suggest that sample concentration increase the diagnostic efficiency of the PMCA assay, in line with previous recommendations ${ }^{10,26}$.

\section{Discussion}

CWD is problematic due to its deadly outcome and its ability to efficiently spread across wild and farmed cervid populations. Sensitive ante-mortem diagnostic assays would be highly useful in identifying early cases of CWD, providing a useful tool for disease management. The development of in vitro prion conversion assays (PMCA ${ }^{10}$ and RT-QuIC ${ }^{11}$ ) provides hope towards the development of ante-mortem CWD tests for easily accessible samples such as blood, feces, urine and saliva ${ }^{19,27,28}$. Additionally, these techniques could be utilized to detect CWD prions longitudinally in experimentally infected WTD using the above mentioned sample types. Blood is an ideal sample type for several reasons: (i) it provides the lowest chances of cross-contamination due to the aseptic nature of collection, (ii) it is routinely taken for other testing on farmed animals, and (iii) it is known to contain seeding competent prions in experimental and natural conditions ${ }^{7,12,29,30}$.

Previous reports show that prion-seeding activity and PrP deposition can be identified in a variety of biological samples from experimentally infected WTD shortly after infection ${ }^{19,28,31}$. Our previously published data ${ }^{12}$ show that we can detect CWD prions, to various degrees, in blood of both early and late pre-symptomatic WTD. That report focused on WTD that were G homozygous at $P R N P$ codon $96^{20}$. We described a mean correct diagnostic identification of $80.6 \%$ in animals known to be CWD positive by IHC, including $100 \%$ specificity, $95.9 \%$ sensitivity in late pre-symptomatic animals, and $52.9 \%$ in early pre-symptomatic animals ${ }^{12}$. However, WTD have different PRNP polymorphisms at codon 96, where GG is considered to be the wild type, and GS and SS animals are less common ${ }^{20}$. Several reports suggest that presence of $S$ at position 96 can modulate susceptibility to prion infection ${ }^{17,18,20}$, as well as peripheral replication and shedding of infectious particles ${ }^{19,20}$. In that sense, polymorphic variations in the prion protein may greatly affect blood-based diagnosis of field samples.

Here, we report diagnostic data using blood from a small cohort of PRNP 96S homozygous and heterozygous WTD. First, we tested whether our WTD PMCA (utilizing PrP 96G susbtrate) was able to replicate CWD prions from animals carrying at least one $96 \mathrm{~S}$ allele. Our results (Fig. 1) shows that this is possible. Surprisingly, our specific WTD PMCA assay replicated brain-derived prions from the terminally ill PrP 96GS animals more efficiently compared to the ones obtained from the brain of a PrP 96GG WTD. Importantly, both sample sources were normalized for their PK-resistance PrP levels before testing them in PMCA. Whether the efficient in vitro prion replication efficiency observed here for PrP 96GS prions is reproducible is part of ongoing studies. One explanation for this outcome could be found in a potential dissimilar proportion of seeding competent prion aggregates compared to the PK-resistant signal they provide. An alternative explanation is that these samples comprise different prion strains with particular (and different) rates of PMCA conversion. Regardless, these results suggest that our PMCA system is suitable for the screening of biological samples derived from WTD carrying PrP 96S alleles.

Considering the results described above, we tested blood samples derived from WTD carrying one or two PrP 96S alleles for their presence of seeding competent CWD prions. The overall diagnostic power for this new WTD cohort substantially decreased when compared to a similar population of animals homozygous for the PrP 96G haplotype ${ }^{12}$. When comparing data of PrP 96GG and PrP 96GS animals, similar sensitivity was observed 
for both animal groups at the early pre-symptomatic stage ( $57.1 \%$ versus $52.9 \%$, respectively). Unfortunately, accurate diagnosis for the late pre-symptomatic PrP 96GS deer reached a similar level of accuracy (58.3\%) when compared to subjects at the early pre-symptomatic phase. These values contrasted with the ones found for the PrP 96GG cohort where correct diagnosis at late pre-symptomatic stages reached $95.9 \%{ }^{12}$. If we consider that just 1 out of $5(20 \%)$ of the CWD-infected PrP 96SS deer was accurately diagnosed, we can assume that the PrP $96 \mathrm{~S}$ polymorphism modulates the presence of CWD prions in peripheral compartments, such as blood, in a dose dependent manner, especially at late stages of the CWD incubation periods. In addition, our data suggest that the presence of CWD prions in peripheral compartments reach their intrinsic maximum levels early during the incubation periods when the PrP 96S allele is present. Future research using controlled experimental conditions may confirm or refute the previously mentioned assumption.

Similarly as described in our previous report ${ }^{12}$, some of the tested samples did not result in positive PMCA detection all the times they were tested. In that sense, our current protocol require several replicates in order to provide accurate results. Additional tests assessing for the reproducibility of our assays were not possible due to the limited amount of sample available. Nevertheless, several optimization studies are currently ongoing in our laboratory using other blood sources and biological specimens. One explanation for the lower detection efficiency in samples from deer carrying at least one PRNP $96 \mathrm{~S}$ allele may be found in potential incompatibilities between the $\operatorname{PrP}^{\mathrm{Sc}} \mathrm{CWD}$ inocula and PMCA substrates. Compelling evidence in several animal species, including WTD, show that polymorphic variation in the prion protein may modulate the susceptibility of disease transmission ${ }^{18,32-34}$. Although the use of PrP 96GG PMCA substrate may have had an effect on our results, the data presented in Fig. 1 suggests that this specific protocol can effectively replicate prions from both PrP 96GS and PrP 96GG origins equally. Unfortunately, PrP 96SS substrate was not available in our laboratory at the moment of running this experiment, and blood samples from WTD available to us were restricted to what was used in this study. This limited further studies using homologous substrate for blood samples derived from PrP 96SS WTD. In addition, we do not know the distribution of each prion allele with respect to the number of $\mathrm{PrP}^{\mathrm{Sc}}$ particles present in brain and blood of infected animals. In that line, the concentration of infectious prion proteins present in peripheral versus brain compartments may be different between PrP 96GG and PrP 96GS animals and could have contributed to the lower diagnostic sensitivity observed in this study. If we additionally consider the possibility that these animals are naturally infected with different strains of the CWD agent (each one of them with specific tropisms to peripheral tissues and PMCA replication properties) the scenario becomes even more complex. Studies in these directions may help to not only further our understanding on natural CWD transmission, but also contribute in refining diagnostic methods.

Our results suggest that blood detection using the current PMCA protocol is limited, especially in early stages of the CWD incubation period. Nevertheless, practices like repeated sampling at different times and improvements in the PMCA protocol (at the sample processing or the prion replication stages), enrichment of certain blood components (e.g., buffy coat), testing PrP polymorphic -specific PMCA substrates, among others, may improve the numbers presented in this article. Another limitation of this study involves the limited sample size interrogated by PMCA. This is more pronounced for the PrP 96 SS samples as just 5 specimens comprising only the early pre-symptomatic stage were tested. In that sense, we acknowledge that further studies utilizing larger cohorts from all polymorphic groups and using complementary techniques (e.g., RT-QuIC) are needed to confirm our findings. The evaluation of CWD-prions content in different blood fractions ${ }^{29}$ for different polymorphic groups may also help not only to improve detection efficiency, but also to provide information on peripheral prion replication for WTD harboring different PrP polymorphic variants. In addition, bioassays would further confirm whether prion infectivity in blood is correlated with in vitro prion detection. Nevertheless, this data suggest that the PRNP codon 96 polymorphisms in WTD influence the ability to detect CWD in blood samples and needs to be considered in advance when pursuing ante-mortem diagnostic tests.

Received: 9 June 2020; Accepted: 16 October 2020

Published online: 13 November 2020

\section{References}

1. Williams, E. S. \& Young, S. Chronic wasting disease of captive mule deer: A spongiform encephalopathy. J. Wildl. Dis. 16, 89-98 (1980).

2. Rivera, N. A., Brandt, A. L., Novakofski, J. E. \& Mateus-Pinilla, N. E. Chronic wasting disease in cervids: Prevalence, impact and management strategies. Vet. Med. Res. Rep. https://doi.org/10.2147/vmrr.s197404 (2019).

3. Benestad, S. L. \& Telling, G. C. Chronic wasting disease: an evolving prion disease of cervids. Handb. Clin. Neurol. https://doi. org/10.1016/B978-0-444-63945-5.00008-8 (2018).

4. Mysterud, A. \& Edmunds, D. R. A review of chronic wasting disease in North America with implications for Europe. Eur. J. Wildl. Res. https://doi.org/10.1007/s10344-019-1260-z (2019).

5. Mateus-Pinilla, N., Weng, H. Y., Ruiz, M. O., Shelton, P. \& Novakofski, J. Evaluation of a wild white-tailed deer population management program for controlling chronic wasting disease in Illinois, 2003-2008. Prev. Vet. Med. https://doi.org/10.1016/j.preve tmed.2013.03.002 (2013)

6. Evans, T. S., Schuler, K. L. \& David Walter, W. Surveillance and monitoring of white-tailed deer for chronic wasting disease in the Northeastern United States. J. Fish Wildl. Manage. https://doi.org/10.3996/032014-JFWM-021 (2014).

7. Mathiason, C. K. et al. Infectious prions in the saliva and blood of deer with chronic wasting disease. Science 314, 133-136 (2006).

8. Tamgüney, G. et al. Asymptomatic deer excrete infectious prions in faeces. Nature 461, 529-532 (2009).

9. Haley, N. J., Seelig, D. M., Zabel, M. D., Telling, G. C. \& Hoover, E. A. Detection of CWD prions in urine and saliva of deer by transgenic mouse bioassay. PLoS ONE 4, e4848 (2009).

10. Morales, R., Duran-Aniotz, C., Diaz-Espinoza, R., Camacho, M. V. \& Soto, C. Protein misfolding cyclic amplification of infectious prions. Nat. Protoc. 7, 1397-1409 (2012). 
11. Schmitz, M. et al. The real-time quaking-induced conversion assay for detection of human prion disease and study of other protein misfolding diseases. Nat. Protoc. https://doi.org/10.1038/nprot.2016.120 (2016).

12. Kramm, C. et al. Detection of prions in blood of cervids at the asymptomatic stage of chronic wasting disease. Sci. Rep. https:// doi.org/10.1038/s41598-017-17090-x (2017).

13. Baylis, M. \& Goldmann, W. The genetics of scrapie in sheep and goats. Curr. Mol. Med. 4, 385-396 (2004).

14. Bruce, M. E. \& Dickinson, A. G. Genetic control of amyloid plaque production and incubation period in scrapie-infected mice. J. Neuropathol. Exp. Neurol. 44, 285-294 (1985).

15. Owen, F., Poulter, M., Collinge, J. \& Crow, T. J. A codon 129 polymorphism in the PRIP gene. Nucleic Acids Res. 18, 3103 (1990).

16. Wadsworth, J. D. F. \& Collinge, J. Molecular pathology of human prion disease. Acta Neuropathol. 121, 69-77 (2011).

17. Johnson, C. et al. Prion protein polymorphisms in white-tailed deer influence susceptibility to chronic wasting disease. J. Gen. Virol. 87, 2109-2114 (2006).

18. Race, B., Meade-White, K., Miller, M. W., Fox, K. A. \& Chesebro, B. In vivo comparison of chronic wasting disease infectivity from deer with variation at prion protein residue 96. J. Virol. 85, 9235-9238 (2011).

19. Plummer, I. H., Wright, S. D., Johnson, C. J., Pedersen, J. A. \& Samuel, M. D. Temporal patterns of chronic wasting disease prion excretion in three cervid species. J. Gen. Virol. 98, 1932-1942 (2017).

20. O'Rourke, K. I. et al. Polymorphisms in the prion precursor functional gene but not the pseudogene are associated with susceptibility to chronic wasting disease in white-tailed deer. J. Gen. Virol. 85, 1339-1346 (2004).

21. Browning, S. R. et al. Transmission of prions from mule deer and elk with chronic wasting disease to transgenic mice expressing cervid PrP. J. Virol. 78, 13345-13350 (2004).

22. Morales, R. et al. Reduction of prion infectivity in packed red blood cells. Biochem. Biophys. Res. Commun. 377, 373-378 (2008).

23. Kramm, C. et al. In vitro detection of chronic wasting disease (CWD) prions in semen and reproductive tissues of white tailed deer bucks (Odocoileus virginianus). PLoS ONE https://doi.org/10.1371/journal.pone.0226560 (2019).

24. Morales, R. et al. Strain-dependent profile of misfolded prion protein aggregates. Sci. Rep. https://doi.org/10.1038/srep20526 (2016).

25. Morales, R., Abid, K. \& Soto, C. The prion strain phenomenon: Molecular basis and unprecedented features. Biochim. Biophys. Acta 1772, 681-691 (2007).

26. Chen, B., Morales, R., Barria, M. A. \& Soto, C. Estimating prion concentration in fluids and tissues by quantitative PMCA. Nat. Methods 7, 519-520 (2010).

27. Mathiason, C. K. et al. Infectious prions in pre-clinical deer and transmission of chronic wasting disease solely by environmental exposure. PLoS ONE 4, e5916 (2009).

28. Henderson, D. M. et al. Longitudinal detection of prion shedding in saliva and urine by chronic wasting disease-infected deer by real-time quaking-induced conversion. J. Virol. 89, 9338-9347 (2015).

29. Mathiason, C. K. et al. B cells and platelets harbor prion infectivity in the blood of deer infected with chronic wasting disease. J. Virol. 84, 5097-5107 (2010).

30. Elder, A. M. et al. In vitro detection of prionemia in TSE-infected cervids and hamsters. PLoS ONE 8, e80203 (2013).

31. Hoover, C. E. et al. Pathways of prion spread during early chronic wasting disease in deer. J. Virol. https://doi.org/10.1128/jvi.00077 -17 (2017).

32. Duque Velásquez, C. et al. Deer prion proteins modulate the emergence and adaptation of chronic wasting disease strains. J. Virol. 89, 12362-12373(2015).

33. Moore, R. C. et al. Mice with gene targetted prion protein alterations show that Prnp, Sinc and Prni are congruent. Nat. Genet. 18, $118-125$ (1998).

34. Mead, S., Lloyd, S. \& Collinge, J. Genetic factors in mammalian prion diseases. Annu. Rev. Genet. https://doi.org/10.1146/annur ev-genet-120213-092352 (2019).

\section{Aknowledgements}

We thank Dr. Thomas Eckland for critical review of the manuscript and suggestions. This work was funded by grant R01AI132695 from the National Institutes of Health to RM and P01AI077774 that covered part of PS salary. The content is solely the responsibility of the authors and does not necessarily represent the official views of the National Institutes of Health or the United States Department of Agriculture.

\section{Author contributions}

C.K. carried out most of the PMCA experiments, analyzed results, and prepared the figures. P.S. contributed in some of the PMCA experiments and helped in the preparation of the figures. T.N. provided the blinded samples of CWD-infected blood. R.M. is the principal investigator and was responsible for coordinating research activity, experimental design, data analysis, funding and writing the manuscript. All authors reviewed and corrected the manuscript.

\section{Competing interests}

$\mathrm{RM}$ is listed as inventor in one patent related to the PMCA technology. The other authors declare no competing interests.

\section{Additional information}

Supplementary information is available for this paper at https://doi.org/10.1038/s41598-020-75681-7.

Correspondence and requests for materials should be addressed to R.M.

Reprints and permissions information is available at www.nature.com/reprints.

Publisher's note Springer Nature remains neutral with regard to jurisdictional claims in published maps and institutional affiliations. 
(c) (i) Open Access This article is licensed under a Creative Commons Attribution 4.0 International cc) License, which permits use, sharing, adaptation, distribution and reproduction in any medium or format, as long as you give appropriate credit to the original author(s) and the source, provide a link to the Creative Commons licence, and indicate if changes were made. The images or other third party material in this article are included in the article's Creative Commons licence, unless indicated otherwise in a credit line to the material. If material is not included in the article's Creative Commons licence and your intended use is not permitted by statutory regulation or exceeds the permitted use, you will need to obtain permission directly from the copyright holder. To view a copy of this licence, visit http://creativecommons.org/licenses/by/4.0/.

(C) The Author(s) 2020 